\title{
Elevated Plasma Levels of Carcinoembryonic Antigen in Cystic Fibrosis
}

\author{
JAMES T. WU, (22) JOHN J. HERBST, AND PATRICK F. BRAY \\ Departments of Pediatrics, Neurology, and Biochemistry, University of Utah Medical Center, \\ Salt Lake City, Utah, USA
}

Extract

The carcinoembryonic antigen (CEA) activity was measured in urine, saliva, and plasma in children with and without cystic fibrosis (CF). Serial determinations of plasma CEA were also made in four of these patients to determine whether plasma CEA level varies with changes in clinical condition. Characterization of CEA-active molecules in the saline extracts of urine, saliva, plasma, and sputum were made with the technique of Sepharose 6B column chromatography. The plasma CEA levels $(4.4 \pm 2.4 \mathrm{ng} / \mathrm{ml})$ in CF children were found to be significantly higher than the reported upper range for normal subjects $(2.5 \mathrm{ng} / \mathrm{ml})$, or those for our control patients of comparable age $1.6 \pm 0.7 \mathrm{ng} / \mathrm{ml}$ ). Good correlation (correlation coefficient 0.87 ) was found between the levels of plasma CEA and Schwachman scores in 22 patients. Parallel changes of plasma CEA levels with changes in the clinical condition were also observed in the follow-up studies. In these four patients the plasma-CEA level was best correlated with the profuseness of rales and rhonchi and the amount of sputum production. Although CEA activity was present in the urine and saliva of CF patients, the CEA concentration did not differ significantly from that of normal control subjects.

Column chromatography was performed on urine, saliva, plasma, and sputum from patients with cystic fibrosis. One heterogenous peak with a molecular weight of approximately 200,000 was noted in all fluids examined and was similar to the CEA peak obtained from colon tumors. Another peak appeared in the void column which corresponded to a molecular weight equal to or greater than 1 million. The latter peak was found in all fluids except plasma. The activity profiles of CEA activity of the physiologic fluids in normal patients and patients with cystic fibrosis were similar.

\section{Speculation}

The correlation of plasma CEA levels with the amount of sputum production in patients with CF may explain the similar elevation of Plasma-CEA in chronic bronchitis, pulmonary tuberculosis, pneumonia, and in smokers. In all of these subjects there is an increase in sputum production. It is also possible that $\mathrm{CEA}$-active glycoproteins in the sputum and in the plasma of patients with cystic fibrosis are disease-associated abnormal glycoproteins. Further investigation may provide new leads to the pathogenesis of cystic fibrosis.

Cystic fibrosis is associated with abnormal mucus production. This abnormality is not only important in the pathogenesis of $C F$ but has also been hypothesized as a basic defect in these children. Although numerous studies have been made to characterize the glycoproteins in this disease, the underlying biochemical defect has not been delineated. In this paper we report our studies on the presence of carcinoembryonic antigenic-active molecules in patients with cystic fibrosis. In 1965. Gold and Freedman (4) identified an oncofetal antigen in extracts of fetal gut and carcinoma of the human digestive tract, and named it carcinoembryonic antigen. The antigen was subsequently identified as a glycoprotein and was found to be localized in the glycocalyx of the plasma membranes of colonic tumor cells (3). Several radioimmunoassays $(8,16)$ have been developed for determining the blood levels of CEA. Large scale collaborative studies $(5,7,8)$ have established recently the blood CEA level as a useful adjunct in the diagnosis and management of the cancer patient. The presence of CEA in normal physiologic fluids and the suggestive evidence that CEA may be identical with an antigen in normal mucous cells $(10-12)$ stimulated us to investigate the presence of this antigen in patients with cystic fibrosis.

CEA activity was measured in urine, saliva (9), and plasma of cystic fibrosis and control children to see whether abnormal levels of this glycoprotein occur in patients with cystic fibrosis. Serial determinations of plasma CEA were made in some patients to determine whether levels varied with changes in clinical condition. Chromatography of CE.A-active molecules in the saline extracts of urine, saliva, plasma, and sputum from patients with cystic fibrosis was performed for the purpose of characterization.

\section{MATERIALS AND METHODS}

Samples of plasma, urine, and saliva were obtained from cystic fibrosis children and control children. Sputum samples were available only from the cystic fibrosis patients. All specimens were refrigerated immediately and the CEA levels were determined within 2 days. Salivary samples were obtained by expectoration of saliva. Only the viscous portion of freshly obtained sputum samples were used in order to minimize salivary contamination. CEA levels in plasma were determined by a zirconium phosphate gel radioimmunoassay. The same procedure was used to determine the CEA levels in the urine and saliva except that pretreatment of the sample with perchloric acid was omitted. Unlike plasma, no protein precipitates occurred after perchloric acid treatment of urine and saliva, and such treatment merely lowers CEA activity. Because of the high activity of CEA in urine and saliva, care was exercised to employ appropriate aliquots so that the results would fall within the range of the standard inhibition curve. These aliquots were always less than the $0.5-\mathrm{ml}$ quantity used in the assay of CEA in plasma. The assay was also modified in that the samples of urine, saliva, and standard CEA were radioimmunoassayed under the same buffer condition $(0.05 \mathrm{M}$ ammonium acetate at $\mathrm{pH}$ 6.5). The procedures for Sepharose 6B column chromatography, creatinine, and protein determinations have all been described in a previous communication (18). Cystic fibrosis was diagnosed on the basis of at least two determinations of elevated sweat chloride and a typical clinical course. The severity of the clinical involvement was established according to the scoring system proposed by Shwachman in which a score of 100 indicates a healthy individual, and scores of less than 50 indicate severe involvement (15). 


\section{RESULTS}

\section{CORRELATION OF PLASMA CEA WITH SEVERITY OF CYSTIC FIBROSIS}

CEA levels in plasma, urine, and saliva from children with and without cystic fibrosis are shown in Table 1. The plasma levels of CEA in cystic fibrosis children were found to be significantly higher than the reported upper range for normal subjects $(2.5$ $\mathrm{ng} / \mathrm{ml}$ ) and for our control patients of comparable age. The significance of the association between $\mathrm{CF}$ and elevated plasma CEA concentration is more apparent when plasma CEA is compared with the Scwachman score of the individual child. Values from 22 children are plotted in Figure 1. The regression line was computed by the method of least square statistical analysis,

Table 1. Carcinoembryonic antigen (CEA) levels in plasma, urine, and saliva of children with and without cystic fibrosis ${ }^{1}$

\begin{tabular}{lccc}
\hline \multirow{3}{*}{ Subjects } & $\begin{array}{c}\text { CEA activity } \\
\text { Plasma, } \\
\text { ng/ml }\end{array}$ & $\begin{array}{c}\text { Urine, ng/mg } \\
\text { creatinine }\end{array}$ & $\begin{array}{c}\text { Saliva, ng/mg } \\
\text { protein }\end{array}$ \\
\hline Cystic fibrosis & $4.4 \pm 2.4(18)^{2}$ & $15 \pm 7.2(18)$ & $143 \pm 131(12)$ \\
Normal & $1.6 \pm 0.7(11)^{2}$ & $23 \pm 1.7(11)$ & $128 \pm 96(8)$
\end{tabular}

${ }^{1}$ Number of children is in parentheses.

${ }^{2} P<0.01$.

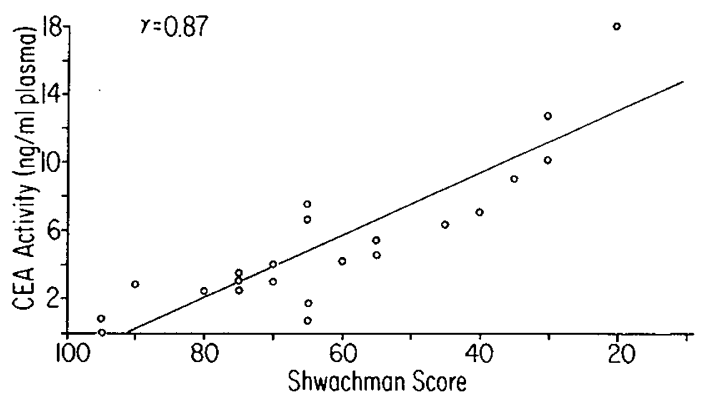

Fig. 1. Correlation between the plasma carcinoembryonic antigen ( $C E A$ ) levels and the severity of the disease (Shwachman score) in children with cystic fibrosis.

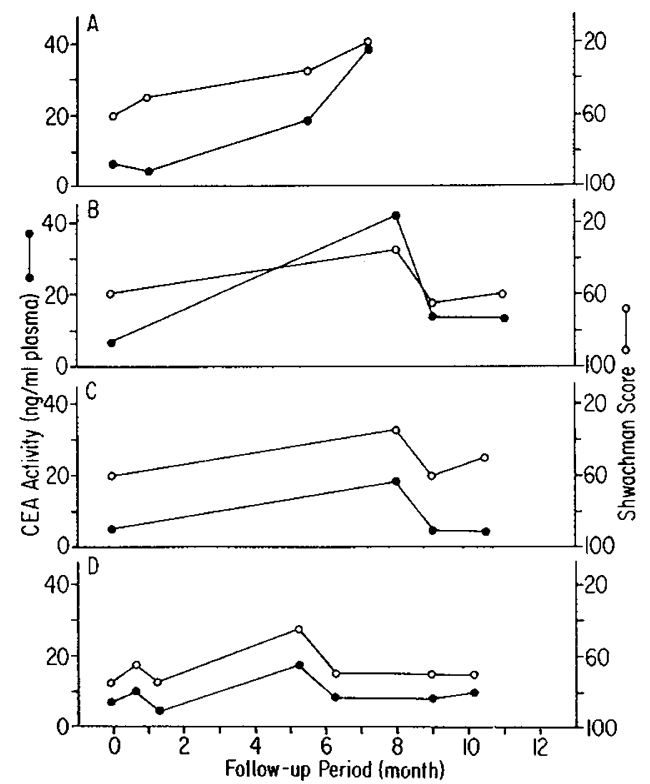

Fig. 2. Sequential measurement of plasma carcinoembryonic antigen $(C E A)$ levels in children with cystic fibrosis. Note the parallel change of plasma CEA levels with the severity of disease in individual children. and the correlation coefficient was 0.87 . The plasma CEA activity of four children with CF who had wide variations in the severity of clinical disease was monitored over a 10 -month period to determine whether plasma CEA levels reflected changes in the severity of the disease. Changes in plasma CEA values and variation in severity of the clinical condition, as reflected in the Schwachman score, are depicted in Figure 2. In each individual, variations in plasma CEA values are associated with a similar change in the Schwachman score. The change in plasma values could be quite rapid; in one patient there was a decrease from 9 to $5 \mathrm{ng} / \mathrm{ml}$ of CEA in 4 days during a time when there was a rapid improvement in the clinical score. The concentration of CEA active molecules in urine and saliva was much higher than in blood (Table 1), but there was no correlation between plasma and urinary or plasma and salivary levels of CEA. Furthermore, no difference was found between $C F$ and control patients in salivary or urinary levels of CEA.

It was observed during the follow-up studies noted above that the plasma CEA levels not only correlated with the severity of the disease as reflected in the Shwachman score but was also correlated with the severity of the lung disease, especially with the profuseness of rales and rhonchi and the amount of sputum production.

\section{COLUMN CHROMATOGRAPHY}

This part of the study was designed to determine whether the CEA-active molecules detected in the body fluids of CF patients differed from CEA derived from colon tumors or CEA-active molecules of the normal physiologic fluids. Samples containing CEA activity were subjected to chromatography on a Sepharose 6B column. For urine and saliva, the clear saline extract of pooled specimens was employed. For plasma, perchloric acid treatment was necessary to remove the majority of the plasma pro-

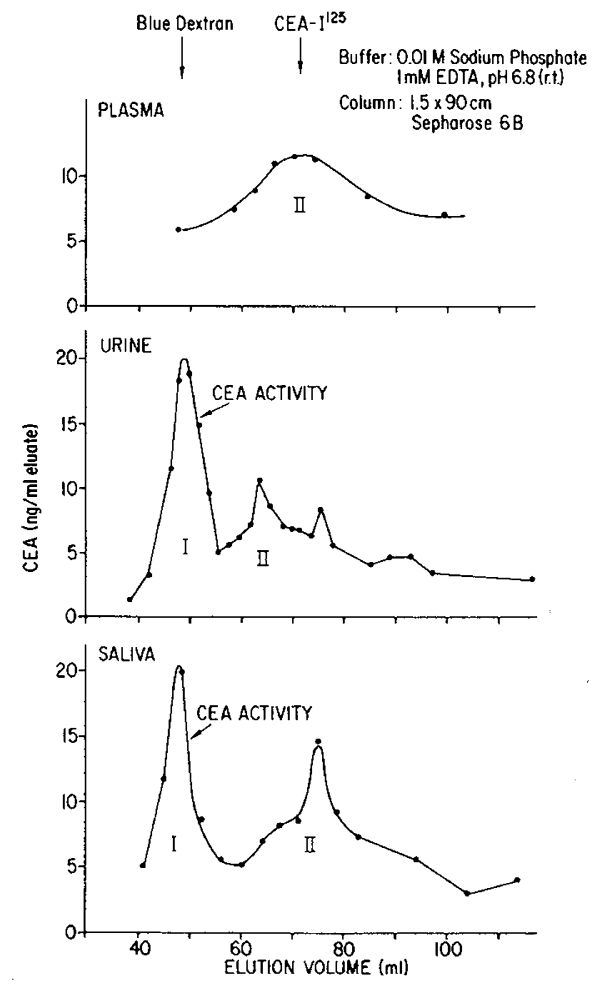

Fig. 3. Column chromatography of plasma, urine, and saliva obtained from children with cystic fibrosis. The arrow on the left indicates the position of the void volume, the arrow on the right indicates the position of $\left[{ }^{125}\right]$ carcinoembryonic antigen $\left(C E A-I^{125}\right)$ provided with a Roche-CEA kit. Column size was 1.5 by $90 \mathrm{~cm}$. The buffer used for column equilibrium and elution was $0.01 \mathrm{M}$ sodium phosphate containing $1 \mathrm{mM}$ EDTA at $\mathrm{pH}$ 6.8 . 
teins before the plasma could be concentrated prior to column application. The results are shown in Figure 3. The CEA activity profiles show no apparent difference from that of normal physiologic fluids (17). Repeated studies consistently showed two groups of CEA activity peaks in the saliva and urine extracts. Group I molecules appeared at the void volume of the column which corresponded to a molecular size equal to or larger than 1 million, and a second heterogenous group of molecules (group $I I$ ) appeared later with molecular weights of approximately 200,000 . In plasma there was only a single CEA activity peak, the size of the molecule being similar to that of colon tumor-CEA, approximately 200,000 .

The sputum from patients with CF was mixed with an equal volume of cold saline and stirred gently at $4^{\circ}$ for approximately $1 \mathrm{hr}$ until it was dispersed into a homogeneous fluid. This fluid was then centrifuged at $27,000 \times g$ for $30 \mathrm{~min}$, and the supernatant fraction was used for CEA measurement and column chromatography. Very high CEA activity and specific activity ( 373 ng CEA/mg protein) were found in sputum saline extracts. The inhibition curve of sputum CEA was parallel with that of the CEA standard. Repeated extractions indicated that the majority of the CEA activity $(75 \%)$ was present in the first extract with very little being solubilized after the third extraction (Table 2). The profile of CEA activity after column chromatography is plotted in Figure 4. Similar to what we have noted in urine and saliva there were again group I and group II CEA activity peaks with molecular weights in the neighborhood of 1 million and 200,000, respectively.

\section{DISCUSSION}

It is now accepted that CEA is neither tumor specific nor of entodermal origin and that the level of plasma CEA may be elevated in various neoplastic diseases as well as in diseases of nonmalignant origin $(5,7)$. The plasma CEA test has been found to be of value in monitoring the effectiveness of therapy and the

Table 2. Extraction of carcinoembryonic antigen (CEA)-active molecule from sputum of cystic fibrosis patients ${ }^{1}$

\begin{tabular}{ccc}
\hline $\begin{array}{c}\text { Successive } \\
\text { extraction }\end{array}$ & $\begin{array}{c}\text { Extracted CEA } \\
\text { activity, ng }\end{array}$ & \% Distribution \\
\hline 1 & 55,800 & 74.7 \\
2 & 10,100 & 13.5 \\
3 & 8,700 & 11.7 \\
4 & 200 & 0.3 \\
\hline
\end{tabular}

${ }^{1}$ Sample of $5 \mathrm{ml} \mathrm{sputum}$ was employed in this study.

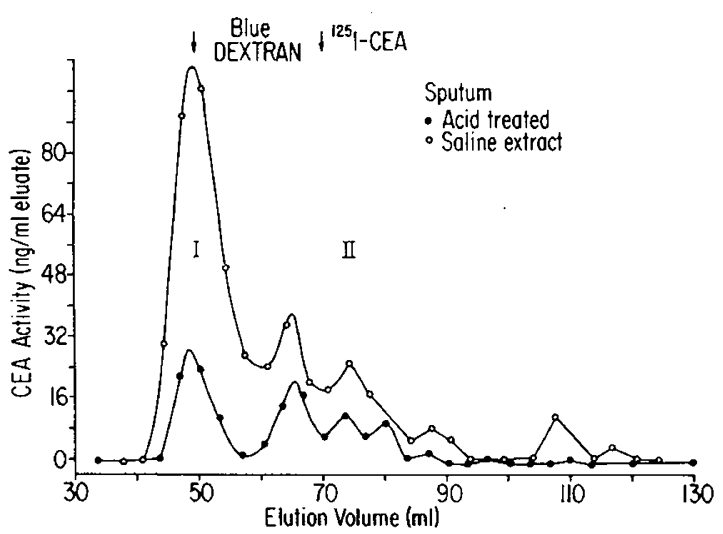

Fig. 4. Column chromatography of saliva extracts of sputum from children with cystic fibrosis with or without treatment with $0.6 \mathrm{M}$ perchloric acid. The condition is the same as we described in Figure 3. Before acid treatment both samples contained the same amount of carcinoembryonic antigen (CEA) activity. The column and the buffer were the same as described in the legend of Figure 3. development of tumor recurrences $(5,8)$. However, it was noticed by Hansen et al. (5) that except for pancreatic and colorectal carcinoma, titers above $20 \mathrm{ng} / \mathrm{ml}$ are rare and are usually associated with the presence of metastatic disease. Our results show that patients with CF, a disease that is not associated with malignancy, can have plasma CEA levels as high as $40 \mathrm{ng} / \mathrm{ml}$. The mechanism whereby plasma CEA becomes elevated in cystic fibrosis is unknown at the present time. However, we have found that the plasma CEA levels in patients with CF are correlated with the severity of the disease. The correlation of the plasma CEA levels with Schwachman score in individuals seems best correlated with the severity of the lung disease and the amount of sputum production in the patient. It is possible that the mechanism for the increase in plasma CEA in cystic fibrosis patients may be similar to the increase noted in chronic bronchitis, pulmonary tuberculosis, pneumonia, and in smokers (5). In all of these conditions there is an increase in mucus production in the lungs, and the CEAactive molecules in the sputum may be transferred into the plasma. The extremely high concentration and specific activity of CEAactive molecules that we have noted in the sputum of CF patients would tend to support this idea. The sputum from CF patients contains the highest concentration of CEA of any body fluid we have examined (17) and is similar to that detected in extracts of colon tumors. The slope of the CEA inhibition curve of sputum indicates sputum CEA has the same high affinity toward anti-CEA antiserum as colon tumor CEA.

Unlike blood plasma, most other human physiologic fluids (17) consistently showed two groups of CEA-active molecules after Sepharose 6B column chromatography. Similar patterns were observed in sputum from patients with cystic fibrosis. Only a single CEA-active peak with molecular weight close to 200,000 was detected in the plasma. If plasma CEA of cystic fibrosis patients is indeed derived from sputum it would be interesting to find out why only the smaller CEA molecules (mol wt approximately 200,000) and not the larger species (mol wt approximately $1,000,000$ ) appeared in the blood. In our previous study of urinary CEA, we failed to demonstrate that the group I CEA-active molecules are an aggregate of the group $I I$ molecules. We are not yet certain whether the conversion of the large molecule to the smaller molecule occurs before it enters the blood. It is also possible that the size of the large molecule simple prevents its entrance into the vascular compartment.

The techniques used in the present study differentiate the CEA-active molecules only according to differences in size. It will be important to determine whether other differences may be present between CEA-active molecules from sputum and plasma of cystic fibrosis patients and those derived from colon tumor.

In addition to CEA-active molecules, which are glycoproteins, many other abnormal proteins and glycoproteins have been noted to be associated with cystic fibrosis. Among these are cystic fibrosis ciliary inhibitory factor found in serum and saliva (18), calcium binding protein in the submaxillary saliva $(1,2)$, and glycoproteins in the sputum of these patients $(6,13,14)$. Comparison between CEA-active molecule and these other glycoproteins in cystic fibrosis deserves further investigation. In the present study we could show only an abnormal increase of CEA-active molecule in the blood but could not demonstrate an abnormal increase in the saliva of cystic fibrosis patients as has been done with the other abnormal glycoproteins.

\section{SUMMARY}

Elevated levels of plasma CEA were found in patients with cystic fibrosis. The elevations were well correlated with the Shwachman scores of the patients. Parallel changes of the levels of plasma CEA and clinical conditions of four patients were also observed in the follow-up studies. Close clinical examination indicated that the elevated plasma CEA of cystic fibrosis patients may be derived from sputum which is produced in abnormal amounts. This hypothesis is further supported by the very high 
content and the chromatographic profile of the CEA activity of sputum from patients with cystic fibrosis.

\section{REFERENCES AND NOTES}

1. Boat, . F. Wiesman, U. N., and Pallavicini, J. C.: Purification and properties of the calcium-precipitable protein in submaxillary saliva of normal and cystic fibrosis subjects. Pediat. Res., 8: 531 (1974).

2. Bowman, B. H., Schmoyer, I., Barnett, D. R., Kurosky, A., and Hutchison, T. Cystic fibrosis-Biochemical characterization of the ciliary inhibitor. Amer. J. Genet., 25: 16A (1973.

3. Gold, R., Krupey, J., and Ansari, H.: Position of the carcinoembryonic antigen of the human digestive system in ultrastructure of tumor cell surface. J. Nat. Cancer Inst., 45: 219 (1970)

4. Gold, P., and Freedman, S. O.: Demonstration of tumor-specific antigens in human colonic carcinomata by immunological tolerance and absorption techniques. J. Exp. Med., I21: 439 (1965).

5. Hansen, J. J., Synder, J. J., Miller, E., Vandevoorde, J. P., Miller, O. H., Hines, L. R., and Burns, J. J.: Carcinoembryonic antigen (CEA) assay. A laboratory adjunct in the diagnosis and management of cancer. Human Pathol., 5: 139 (1974).

6. Lamblin, G., Degand, P., Roussel, P., Havez, R., Harteman, E., and Fillot, M.: Glycopeptides of bronchial fibrillar mucous in cystic fibrosis. Clin. Chem. Acta, 36: 329 (1972)

7. Laurence, D. J. R. Stevens, U., Bettelheim, R., Daray, D., Leese, C., Turberville, C., Alexander, P., Johns, E. W., and Neville, A. M.: Role of plasma, carcinoembryonic antigen in diagnosis of gastrointestinal, mannary and bronchial carcinoma. Brit. Med. J., 3: 605 (1972).

8. LoGerfo, P., Krupey, J., and Hansen, H. J.: Demonstration of an antigen common to several varieties of neoplasia. N. Engl. J. Med., 285: 138 (1971).

9. Martin, F., and Devant $\mathrm{J}$.: Carcinoembryonic antigen in normal human saliva. J. Nat. Can. Inst., 50: 1375 (1974).

10. Rogalsky, V. Y., and Konovich, E. A.: Localization of organ antigens in the human large intestine. Bull. Exp. Biol., 12: 64 (1971).

11. Rogalsky, V. Y.: Identity of carcino-embryonal antigen and antigen of mucousproducing cells. Lancet, $i: 1322$ (1973).

12. Rogalsky, V. Y.: Tumor-cell mucous protein and CEA. Lancet, ii: 729 (1974).

13. Roussel, E. Lamblin, G., Degan, P., and Havez, R.: Isolation of bronchial mucins secreated in cystic fibrosis. Clin. Chim. Acta, 36: 315 (1972)

14. Roussel, P., Lamblin, F., Degand, P., Walker-Masir, E., and Jeanlz, R. W. Heterogeneity of the carbohydrate chains of sulfated bronchial glycoprotein isolated from a patient suffering from cystic fibrosis. J. Biol. Chem., 250 2114 (1975)

15. Shwachman, H., and Kulczycki, L. L.: Long term study of one hundred five patients with cystic fibrosis-Studies made over a five to fourteen year period. Amer. J. Dis. Child., 96: 6 (1958).

16. Thomson, M. P., Kurpey, J., Freedman, S. O., and Gold, P.: The radioimmunoassay of circulating carcinoembryonic antigen of the human digestive system. Proc. Nat. Acad. Sci. U. S. A., 64: 161 (1969).

17. Wu, J. T., Herbst, J. J., and Bray, P. F.: Unpublished data; Manuscript in preparation

18. Wu, J. T., Madsen, A., and Bray, P. F.: Quantitative measurement and chromatography of human urinary carcinoembryonic antigen activity. J. Nat. Can. Inst., 53: 1589 (1974).

19. The authors wish to express their appreciation for the technical help of Mr. Ace Madsen and Miss Debbi Brown.

20. This research was supported by a grant from the National Cystic Fibrosis Research Foundation, Public Health Service Training Grants 2T 1-NS5503 and 5Ti-NS-5309 from the National Institute of Neurological Diseases and Stroke; Grant 73-0263 from Utah State Division of Health; small institutional gran from the American Cancer Society, University of Utah, and the Eleanor Roosevelt Cancer Research Foundation.

21. A portion of the results was presented at the 15th Annual Meeting for the Society of Pediatric Research, April 30, 1974 in Washington, D.C.

22. Requests for reprints should be addressed to: J. T. Wu, Ph.D., Division of Pediatric Neurology, University of Utah Medical Center, Salt Lake City, Utah 84132 (USA).

23. Accepted for publication November 17, 1975
Hemagglutination inhibition antibody influenza $\mathrm{A}$ virus intranasal local antibody recombinant virus serum antineuraminidase antibody vaccine, live

\title{
Temperature-sensitive Mutants of Influenza A Virus: Response of Children to the Influenza A/Hong Kong/68-ts-1[E] (H3N2) and Influenza A/Udorn/72-ts-1[E] (H3N2) Candidate Vaccine Viruses and Significance of Immunity to Neuraminidase Antigen
}

\author{
HYUN WHA KIM, JULITA O. ARROBIO, CARL D. BRANDT, ROBERT H. PARROTT, ${ }^{(29)}$ \\ BRIAN R. MURPHY, DOUGLAS D. RICHMAN, AND ROBERT M. CHANOCK \\ Research Foundation of Children's Hospital National Medical Center, Department of Child Health and \\ Development, George Washington University School of Medicine, Washington, D.C., and the Laboratory of \\ Infectious Diseases National Institute of Allergy and Infectious Diseases, National Institutes of Health,
} Bethesda, Maryland, USA

\section{Extract}

The influenza A/Hong Kong/1968-ts-1[E] (H3N2) candidate live virus vaccine strain, which had previously been shown to be safe and protective in seronegative adult volunteers, was administered intranasally to 21 children at a dose of $10^{5}$ TCID $_{50}$. One group contained 15 children (5-11 years of age) who lacked serum anti- body to the hemagglutinin $(\leq 1: 8)$, but possessed serum antibody to the neuraminidase antigen. The second group included six children $(24 / 12-31 \% 12$ years of age) who lacked serum antibody to both hemagglutinin and neuraminidase surface antigens of the influenza A virus. Twelve of the 15 children in the first group were infected, but only one child developed mild rhinitis; 6 of the 12 infected vaccinees shed virus for a short interval, while 11 of the group developed 